\title{
Efecto del tabaquismo durante el embarazo sobre la antropometría al nacimiento
}

Luisa María Sánchez-Zamorano, MVZ, M en C, ${ }^{(1,3)}$ Martha María Téllez-Rojo, Lic, M en C, D r en C, ${ }^{(2,3)}$ Mauricio Hernández-Avila, MD, M PH, PhD.(4)

\section{Sánchez-Zamorano LM,Téllez-Rojo MM, Hernández-Avila M. \\ Efecto del tabaquismo durante el embarazo sobre la antropometría al nacimiento. Salud Publica Mex 2004;46:529-533. El texto completo en inglés de este artículo está disponible en: http://www.insp.mx/salud/46/eng}

\section{Resumen}

Objetivo. Evaluar el efecto del consumo de tabaco durante el embarazo sobre el peso y la longitud al nacimiento. Ma terial y métodos. Mediante el análisis secundario de una base de datos de dos estudios de cohorte, realizados en la Ciudad de México de 1993 a 2000 sobre exposición a plomo, se evaluaron las diferencias en el peso y longitud al nacer entre los hijos de mujeres que refirieron haber fumado durante el embarazo y las que no lo hicieron. Las diferencias se estimaron ajustando por otros factores importantes para el peso y la longitud al nacer, mediante modelos de regresión lineal múltiple. Resultados. La proporción de mujeres que dijeron fumar durante el embarazo fue de $4.7 \%$. Se observó que los hijos de madres que fumaron durante el embarazo pesaron en promedio 154 gramos menos (IC 95\% -284.7 -24.7), y midieron 0.79 centímetros menos (IC 95\% $1.5-0.1)$, en comparación con los hijos de mujeres que refirieron no haber fumado durante el embarazo. Conclusiones. Estos hallazgos ponen en evidencia el daño producido por el consumo de tabaco sobre la población infantil. El texto completo en inglés de este artículo está disponible en: http://www.insp.mx/salud/46/eng

Palabras clave: recién nacido; tabaquismo; embarazo; peso al nacer; longitud al nacer; México

\author{
Sánchez-Zamorano LM,Téllez-Rojo MM, \\ Hernández-Avila M. \\ Effect of smoking during pregnancy \\ on anthropometric characteristics at birth. \\ Salud Publica Mex 2004;46:529-533. \\ The English version of this paper \\ is available at: http://www.insp. $\mathrm{mx} / \mathrm{salud} / \mathbf{4 6} / \mathrm{eng}$
}

\begin{abstract}
A bstract
Objective. To assess the effect of tobacco consumption during pregnancy on height and birth weight of newborns. Material and Methods Two cohort studies on lead exposure conducted in Mexico C ity between 1993 and 2000 were analyzed. $D$ ata were analyzed to assess the differences in height and birth weight between children of women who smoked during pregnancy and those who did not, adjusting for other important factors. Statistical analysis consisted of multiple linear regression models. Results The proportion of women who smoked during pregnancy was $4.7 \%$. The birth weight of children of mothers who smoked during pregnancy was 154 grams less on average (95\% confidence interval [ $\mathrm{Cl}]-284.7,-24.7)$ and their height was 0.79 $\mathrm{cm}$ less $(95 \% \mathrm{Cl}-1.5,-0.1)$ than the children of non-smokers during pregnancy. Conclusions These findings evidence the deleterious effect of tobacco consumption on children. The English version of this paper is available at: http:// www.insp. mx/salud/46/eng
\end{abstract}

Key words: infant, newborn; smoking; pregnancy; birth weigth, birth height; Mexico

(1) Departamento de Investigación sobre Tabaco, D irección de Enfermedades Crónicas, Instituto N acional de Salud Pública (IN SP), Cuernavaca, Morelos, México.

(2) Dirección de Salud Ambiental, IN SP, Cuernavaca, Morelos, México.

(3) Centro de Investigación en Salud Poblacional, IN SP, Cuernavaca, Morelos, México.

(4) Dirección General del IN SP, Cuernavaca, Morelos, México.

Fecha de recibido: 29 de marzo de 2004 - Fecha de aprobado: 6 de agosto de 2004

Solicitud de sobretiros: Luisa María Sánchez Zamorano, D epartamento de Investigación sobre Tabaco, Dirección de Enfermedades Crónicas, Centro de Investigación en Salud Poblacional, Instituto N acional de Salud Pública. Avenida Universidad 655, colonia Santa María A huacatitlán 62508, Cuernavaca, Morelos, México.

Correo electrónico: szamoran@ correo.insp.mx 
S e ha informado que el efecto del humo de tabaco $\mathcal{S}$ de madres que fumaron o estuvieron expuestas a éste durante la gestación puede producir la disminución del peso y la longitud del recién nacido. ${ }^{1-9}$ También se ha observado que fumar durante la gestación, aumenta el riesgo de abortos y de mortalidad neonatal. ${ }^{10}$ En México, la proporción de mujeres embarazadas que han fumado es de $8.0 \%$, lo que representa un valor aproximado de la exposición in utero de los recién nacidos. ${ }^{11}$ Esta exposición in utero a los componentes del humo del tabaco se ha asociado con la mortalidad posnatal; esto se sustenta en el hecho de que en los países con baja tasa de mortalidad posnatal la prevalencia de bajo peso es menor a $10 \%$, mientras que en aquellos países con elevada prevalencia de nacimientos de niños con bajo peso aumenta la tasa de mortalidad posnatal. ${ }^{12}$ Asimismo, los infantes que nacen con bajo peso, de acuerdo con su edad gestacional, son más propensos a enfermar, ya que se ha observado mayor número de días de hospitalización en niños que nacieron con bajo peso para su edad gestacional, en comparación con aquellos que nacieron con peso adecuado. ${ }^{13}$ Otro efecto reportado sobre la exposición in utero al humo del cigarro es que los fetos expuestos a éste tienen menor volumen pulmonar al nacimiento, así como incremento del volumen del intersticio en el parénquima pulmonar. ${ }^{14-16} \mathrm{El}$ feto, al tener que adaptarse a una limitación de desarrollo sufre cambios en su estructura y metabolismo, así como falta de maduración de algunos órganos y sistemas; estas alteraciones pueden ocasionar que en etapa tardía de la vida pueda desarrollar enfermedades degenerativas. ${ }^{17}$

En México existen pocos datos específicos sobre el efecto del tabaquismo durante el embarazo, ${ }_{1}^{11}$ y por ello el objetivo del presente trabajo fue evaluar la posible asociación de fumar durante el embarazo con el decremento en el desarrollo fetal, definido a partir del peso y la longitud del recién nacido.

\section{Material y métodos}

\section{Diseño}

Se realizó un análisis secundario de una base de datos de 793 mujeres embarazadas que participaron en dos estudios de cohorte realizados en la Ciudad de México de 1993 a 2000 sobre exposición a plomo. La recolección de la información sobre las características maternas se hizo durante la última etapa pre-embarazo, la información de tabaquismo activo se recopiló durante el periodo de gestación, y las variables del recién nacido se obtuvieron al momento del parto. El objetivo principal del estudio global de donde parte este trabajo fue el de establecer las relaciones entre diferentes parámetros ambientales y la salud del neonato, y su posterior desarrollo cognoscitivo. El objetivo de este estudio fue el de analizar la contribución del tabaquismo materno durante la gestación y su efecto en la antropometría del recien nacido. La información sobre salud, así como de las características sociales, demográficas y ambientales de las madres y sus productos se recolectó mediante la aplicación de cuestionarios. Personal de las salas de tococirugía de los hospitales tomó las medidas antropométricas del neonato dentro de las primeras 12 horas después del nacimiento. Los métodos han sido descritos con detalle en otras publicaciones. ${ }^{18,19}$

Los criterios de inclusión para las cohortes fueron: que las mujeres estuvieran en edad reproductiva, sin problemas clínicos como diabetes, hipertensión arterial, o padecer de sus facultades mentales, que pensaran permanecer por lo menos un año en la Ciudad de México, que tuvieran el antecedente de haber tenido gestaciones o embarazos simples, y que aceptaran firmar una carta de consentimiento informado.

\section{A nálisis de la información}

Las variables de interés fueron el peso (gr) y la longitud $(\mathrm{cm})$ del recién nacido. Se definió la exposición de tabaquismo durante la gestación en aquellas mujeres que reconocieron haber fumado en cualquier periodo de la gestación. Se realizó el análisis de la información del tabaquismo materno durante la gestación en relación con las medidas antropométricas de sus respectivos bebés, al momento del nacimiento. Previa verificación de los supuestos teóricos correspondientes, el análisis de las variables continuas se hizo mediante la prueba de $t$ de Student; las variables categóricas, por la prueba exacta de Fisher. Se realizaron modelos de regresión lineal múltiple para evaluar el efecto del tabaquismo materno durante la gestación en el peso y la longitud del recién nacido. Estos modelos se ajustaron por la edad gestacional, sexo del recién nacido, peso y edad de la madre. Los supuestos de los modelos de regresión lineal propuestos fueron evaluados con técnicas de diagnóstico estadístico estándar, que consistieron en el análisis de los residuales, evaluación de la multicolinealidad e identificación de valores influyentes.

\section{Resultados}

Se analizó la información correspondiente a 793 mujeres y sus recién nacidos durante el periodo de 1993 a 2000. La mediana de edad de las madres fue de 25 años, 
con un rango intercuartil (RIQ) de 25 a 29. La mediana de paridad previa, fue dos hijos (RIQ 1-3). El porcentaje de mujeres que fumaron en algún momento durante la gestación fue de $4.7 \%$, de las cuales $71.4 \%$ fumaba menos de tres cigarros diariamente. Del total de nacimientos analizados, $45 \%$ fueron niñas y $55 \%$ niños (cuadro I). Se detectó una diferencia significativa en el peso y la longitud por sexo en los recién nacidos (niños $=3$ 169 gr vs. niñas $=3073 p<0.01)$ y (niños $=50.5 \mathrm{~cm}$ vs. niñas $=49.9 \mathrm{~cm} p<0.05)$, respectivamente (datos no mostrados).

El modelo de regresión lineal múltiple, después de ajustar por sexo del recién nacido, edad gestacional, peso de la madre antes de la gestación y número

\section{Cuadro I \\ DistribuCIÓN DE LAS VARIABLES DEL RECIÉN NACIDO ASÍ COMO DE LA MADRE EN LA POBLACIÓN DE ESTUDIO de la Ciudad de México, 1993-2000}

\begin{tabular}{lrrr} 
Variable & $n$ & Media & DE \\
Peso al nacimiento $(\mathrm{gr})$ & 793 & 3125.76 & 441.59 \\
\hline Longitud del bebe al nacimiento $(\mathrm{cm})$ & 789 & 50.22 & 2.40 \\
\hline Edad gestacional (semanas) & 793 & 39.01 & 1.66 \\
\hline Número de gestaciones & 793 & 2.02 & 1.17 \\
\hline Peso de la madre antes de la gestación $(\mathrm{kg})$ & 793 & 66.02 & 10.16 \\
\hline Edad de la madre (años) & 789 & 25.26 & 5.29 \\
& & & \\
$\begin{array}{l}\text { Prematuro (<37 sem) } \\
\quad \text { N o }\end{array}$ & & & \\
\hline Sí & 732 & 92.89 & \\
\hline
\end{tabular}

Tabaquismo durante la gestación

\begin{tabular}{lrr} 
No & 756 & 95.33 \\
\hline Sí & 37 & 4.67
\end{tabular}

Edad de la madre

\begin{tabular}{rrr}
$14-25$ & 444 & 56.27 \\
\hline $26-35$ & 310 & 39.29 \\
\hline $36-44$ & 35 & 4.44
\end{tabular}

Bajo peso $(<2500 \mathrm{~kg})$

\begin{tabular}{lrr} 
No & 749 & 94.45 \\
\hline Sí & 44 & 5.55
\end{tabular}

Sexo del bebé

\begin{tabular}{lll} 
N iña & 361 & 45.22 \\
\hline N iño & 432 & 54.48
\end{tabular}

DE = D esviación estándar de gestaciones, evidenció una asociación negativa estadísticamente significativa entre el tabaquismo durante el embarazo y el peso al nacer. En comparación con las madres que no fumaron durante el embarazo, para aquellas que sí lo hicieron sus recién nacidos pesaron en promedio 154 grs menos (cuadro II). El análisis de regresión lineal cumple con los supuestos de esta metodología estadística, al presentar una distribución normal de los residuos normales y estandarizados (Prueba de Shapiro-Wilk $p>0.05$ ), no presentar problemas de heterocedasticidad (Prueba de Cook-Weisburg $p=0.92)$, y sin presencia de colinealidad.

El modelo correspondiente a la longitud al nacimiento se ajustó por las mismas variables utilizadas en el modelo para peso al nacimiento, además de la variable dicotómica de bajo peso. Este modelo estima que, en promedio, los recién nacidos de mujeres que fumaron en algún periodo de la gestación tuvieron una longitud al nacer de $79 \mathrm{~mm}$ menos que aquellos recién nacidos de madres que no lo hicieron, y el modelo no presentó problemas de heterocedasticidad (Prueba de Cook-Weisburg $p=0.24$ ), ni presentó colinealidad.

Se hizo el análisis de la posible modificación del efecto del sexo con los dos eventos estudiados, no encontrándose una asociación significativa para ambas (peso al nacer, $p=0.491$, longitud al nacimiento, $p=$ 0.951). (Datos no mostrados).

\section{Discusión}

En el presente estudio pudimos evaluar el efecto del consumo materno de tabaco en el desarrollo fetal. El tamaño de la muestra permitió estimar este efecto aun con el bajo porcentaje de mujeres que fumaron durante la gestación (4.7\%), el cual fue ligeramente mayor a la mitad de lo que reportaron en el estudio hecho en el occidente de nuestro país. ${ }^{11}$ Nuestros resultados son similares a otros estudios en donde se ha observado que aquellos recién nacidos de madres fumadoras tienen en promedio 200 gr menos que los recién nacidos de madres no fumadoras. ${ }^{20-24}$ Se ha observado que a mayor número de cigarros fumados durante la gestación, en promedio, el peso al nacimiento del bebé disminuye, sobre todo en aquellas que fumaron 10 o más cigarros al día. ${ }^{25}$

Los efectos que tiene el humo del tabaco sobre el feto son diversos, y algunos autores han sugerido que el monóxido de carbono, al ser absorbido por el feto produce un aumento en la carboxyhemoglobina fetal. ${ }^{17}$ La nicotina proveniente del tabaco consumido por la madre aumenta la tasa cardiaca fetal, principalmente en el segundo trimestre de gestación, debido a una activación del sistema simpático. En el tercer trimestre 
hacían; pero esta información fue obtenida durante el embarazo, por lo tanto no fue condicionada por el proceso del parto, por lo que estaríamos obteniendo una subestimación del efecto; sin embargo, nuestros resultados son similares a los hallados por otros autores..$^{20-24}$

Por lo tanto, concluimos que los hallazgos de este estudio apoyan la evidencia del daño que se produce a la salud de los recién nacidos porque sus madres fumaron durante el embarazo, sobre todo en una generación de mujeres que ha estado expuesta a la información sobre los daños que puede producir el tabaquismo, pero que aún así continúan fumando durante la gestación. Esta información es importante en la perspectiva de mejorar los programas encaminados a un desarrollo saludable de la población infantil en México.

\section{Agradecimientos}

A la licenciada Maritza Solano González, por su desinteresado apoyo en la conformación de la base de datos y revisión del manuscrito.

\section{Referencias}

1. D avies DP, G ray O P, Ellwood PC, A bernethy M. Cigarette smoking in pregnancy:Associations with maternal weight gain and fetal growth. Lancet 1976; 21:385-387.

2. Bosley ARJ, Sibert JR, N ewcombe RG. Effects of maternal smoking on fetal growth and nutrition.Arch D is Child 1981; 56:727-729.

3. Harrison CG, Branson RS, Vaucher YE. Association of maternal smoking with body composition of the newborn. Am J C lin N utr 1983; 38:757-762

4. Cliver SP, G oldenberg RL, C utter GR, H offman HJ, D avis RO, N elson $K G$. The effects of cigarette smoking on neonatal anthropometric measurements. 0 bstet Gynecol 1995; 85:625-630.

5. Roquer JM, Figueras J, Botet J, Jiménez R. Influence on fetal growth of exposure to tobacco smoke during pregnancy.Act Paediatr 1995; 84:118-121.

6. Rubin DH, Krasilnikoff PA, Leventhal JM, W eile B, Berget A. Effect of passive smoking on birth-weight. Lancet 1986; 23:415-417.

7. Luciano A, Bolognani M, Biondani P, G hizzi C, Zoppi G, Signori E. The influence of maternal passive and lignt active smoking on intrauterine growth and body composition of the newborn. Eur J C lin N utr 1998 0 ct;52(10):760-763.

8. Byrd RS, Howard CR. Children's passive and prenatal exposure to cigarette smoke. Pediatric Annals 1995;24:640-645.
9. Lackmann GM, Salzberger U,Töllner U, Chen M, C armella SG, Hecht SS. Metabolites of a tobacco-specific carcinogen in urine from newborns.J N atl C ancer Inst 1999; 91(5):459-465

10.W isborg K, Kesmodel U, Henriksen TB, 0 Isen SJ, Secher N J. Exposure to tobacco smoke in utero and the risk of stillbirth and death in the first year of life. Am J Epidemiol 2001; 154;322-327.

11. Frank R, Pelcastre B, Salgado de Snyder VN , Frisbie W P, Potter JE, Bronfman-Pertzovsky MN . Low birth weight in Mexico: $\mathrm{N}$ ew evidence from a multi-site postpartum hospital survey. Salud Publica Mex 2004;46:23-31.

12. A shworth $A$. Effects of intrauterine growth retardation on mortality and morbidity in infants and young children. Eur J C lin N utr 1998 Jan; 52 suppl 1:S34-S41.

13.Yoshida-Ando P, Mendoza-Pérez AM. Estudios sobre recién nacidos de bajo peso al nacimiento y su seguimiento longitudinal. Salud Publica Mex 1988; 30:25-42.

14. Collins MH, Moessinger AC, Kleinerman J, Bassi J, Rosso P, Collins $A M$ et al. Fetal lung hypoplasia associated with maternal smoking:A morphometric analisis. Pediatr Res 1985; 19:408-412.

15. Lichtenbeld $H$, Vidic B. Effect of maternal exposure to smoke on gas diffucion capacity in neonatal rat. Respir Physiol 1989; 75:129-140.

16. Hanspeter W, Joad JP, Pinkerton KE. The toxicology of environmental tobacco smoke.Annu Rev Pharmacol Toxicol 1997; 37:29-52.

17. C unningham FG, MacD onald PC, G ant N F, Leveno KJ, Gilstrap LC, Hankins GDV et al. 0 bstetricia. 20a. Ed. Editorial Médica Panamericana; 2000:47-89.

18. G onzález-C ossío T, Sanín LH, H ernández-A vila M, Rivera J, Hu H. Longitud y peso al nacer: el papel de la nutrición materna. Salud Publica Mex 1998;40;119-126.

19.Tellez-Rojo MM, Hernández-Avila M, González-Cossío T, Romieu I, A ro A, Palazuelos E et al. Impact of breastfeeding on the mobilization of lead from bone. Am J Epidemiol 2002;155:420-428.

20. Picone TA, Allen LH, O Isen PN , Ferris ME. Pregnancy outcome in $\mathrm{N}$ orth American women. II Effects of diet, cigarette smoking, stress, and weight gain on placentas an on neonatal physical and behavioral characteristic. Am J C lin N utr 1982; 36:1214-1224.

21. N aeye RL. Effects of cigarette smoking on the fetus and neonate. In Disorders of the placenta, fetus and neonate. St Loius: Mosby year book; 1992:88.

22. Economides D, Braithwaite J. Smoking, pregnancy and the fetus. J Roy Soc Health 1994; 114:198-201.

23. MacD orman MF, C nattingius S, Hoffman HJ, D ramer MS, Haglund B. Sudden infant death syndrome and smoking in the United States and Sweden. Am J Epidemiol 1997; 146:249-255.

24. Shiverick KT, Salafia C. Cigarette smoking and pregnancy I: 0 varian, uterine and placental effects. Placenta 1999; 20:265-272.

25. England LJ, Kendrick JS, Gargiullo PM, Zahniser SC, Hannon W H. Measures of maternal tobacco exposure and infant birth weight at term. Am J Epidemiol 2001;153:954-960.

26. Rama-Sastry BV, Chance MV, Hemontolor ME, Goddijn-W essel TAW. Formation and retention of cotinine during placental transfer of nicotine in human placental cotyledon. Pharmacology 1998; 57:104-116. 27. Surgeon $G$ eneral. The health consequences of smoking: N icotine addiction, A ppendix B. U.S. D epartment of health and human services. Atlanta (GA): CDC; 1988:589-618. 